Recepción: 07 / 08 / 2016

Aceptación: 31 / 01 / 2017

Ciencias de la educación Articulo de investigación

Publicación: 06 / 03 / 2017

\title{
El papel de la Universidad en la gestión de la Diversidad
}

\author{
The role of the University in the management of Diversity
}

O papel da Universidade na gestão da Diversidade

\author{
Nancy L. Reyes-Mero ${ }^{\mathrm{I}}$ \\ nancyfj360@ hotmail.com \\ Marjorie M. Gómez-Zambrano ${ }^{\text {II }}$ \\ marjorie.gomez@hotmail.com \\ Patricia J. López-Mero ${ }^{\text {III }}$ \\ pattylopez@hotmail.com RichardF. \\ Rodríguez-Andrade ${ }^{\mathrm{IV}}$ \\ richard.rodriguez@uleam.edu.ec
}

Correspondencia: nancyfj360@ hotmail.com

\footnotetext{
${ }^{\text {I }}$ Magister en Trabajo Social, Licenciada en Trabajo Social, Trabajadora Social, Docente de la Universidad Laica Eloy Alfaro de Manabí, Manta, Ecuador.

II Magister en Gerencia Educativa, Diplomado Superior en Mediación, Licenciada en Trabajo Social, Trabajadora Social, Docente de la Universidad Laica Eloy Alfaro de Manabí, Manta, Ecuador.

${ }^{\text {III }}$ Magister en Gerencia Educativa, Diploma Superior en Intervención Social, Licenciada en Trabajo Social Especialidad Atención a la familia y al desarrollo comunitario, Licenciada en Ciencias de la Educación Especialidad Castellano y Literatura, Profesora de Segunda Enseñanza Especialidad Castellano y Literatura, Docente de la Universidad Laica Eloy Alfaro de Manabí, Manta, Ecuador.

Iv Magister en Gerencia Educativa, Licenciado en Ciencias de la Educación Especialización Docencia Técnica en Informática, Analista de Sistema, Profesor de Educación Pre-Primaria- Nivel Técnico Superior, Docente de la Universidad Laica Eloy Alfaro de Manabí, Manta, Ecuador.
} 


\section{Resumen}

Actualmente la diversidad enfrenta a la universidad a situaciones que parecer tender a obviar el crecimiento real de los estudiantes, las instituciones universitarias tradicionalistas se fundamentaba en un pretendido modelo único de educación al que debían adaptarse los estudiantes independientemente de su procedencia, modelo que entró en decadencia por su cuestionamiento social y por no ofrecer a los estudiantes alternativas efectivas para su adaptación a este medio tan complejo como lo es el medio universitario. La educación como derecho universal, constituye un proceso para el desarrollo de la dignificación y felicidad del ser humano, es por ello que la educación como un sistema establece en sus leyes y normativas la garantía por parte del estado de una educación de calidad para todos, permanente, continua, integral, con equidad de género, igualdad de condiciones y oportunidades, derechos y deberes. Es importante destacar que uno de los aspectos importantes en el sistema educativo es la prosecución de los estudios, esto quiere decir que el estado debe proporcionar la estructura necesaria, no solo para el ingreso en este sino para su mantenimiento prolongado que les permita a las personas independientemente de su raza, sexo o credo alcanzar las metas propuestas en el pleno desarrollo de los individuos como profesionales, logrando así su autorrealización. Así mismo, se hace necesario señalar que las instituciones universitarias iniciaron su filiación histórica en el Medioevo europeo y, a través de rupturas y continuidades, han mantenido una legitimidad social hasta el presente. Su importancia es relevante, al punto de generar expectativas y debates por su estado presente y las potencialidades de su evolución futura. Las universidades han existido desde antes que el estado nacional y seguramente sobrevivirá hasta nuestros días, ya que la historia de la institución universitaria se despliega en la larga duración, desde la que se vislumbra la transferencia y evolución de los modelos más importantes del mundo moderno, así como, sus procesos de transformación y las tendencias de su evolución a futuro. De allí que, se hace importante considerar el papel que estas instituciones les corresponde desempeñar en lo que respecta sus políticas de ingreso y atención a la diversidad, para que la sociedad en general y de manera particular los jóvenes cuyas competencias le permitan acceder a las instituciones universitarias puedan hacerlo y cumplir con los pensum de estudios respectivos con la finalidad de alcanzar sus metas y lograr su autorrealización.

Palabras clave: diversidad; educación superior; universidad 


\section{Abstract}

Currently diversity confronts the university to situations that seem to tend to obviate the real growth of students, the traditional university institutions was based on a pretended unique model of education to which students should adapt regardless of their origin, model that went into decline for their social questioning and for not offering students effective alternatives for their adaptation to this complex environment as is the university environment. Education as a universal right is a process for the development of the dignity and happiness of the human being, that is why education as a system establishes in its laws and regulations the guarantee by the state of a quality education for all, permanent, continuous, integral, with gender equity, equality of conditions and opportunities, rights and duties. It is important to emphasize that one of the important aspects in the education system is the continuation of studies, this means that the state must provide the necessary structure, not only for the entrance in this but for its prolonged maintenance that allows people Regardless of their race, sex or creed reach the goals proposed in the full development of individuals as professionals, thus achieving self-realization. Likewise, it is necessary to point out that the university institutions began their historical affiliation in the European Middle East and, through ruptures and continuities, they have maintained a social legitimacy up to the present. Its importance is relevant, to the point of generating expectations and debates due to its present state and the potentialities of its future evolution. Universities have existed since before the national state and will surely survive to this day, since the history of the university institution unfolds in the long term, from which the transfer and evolution of the most important models of the modern world can be glimpsed, as well as, its transformation processes and the trends of its future evolution. Hence, it is important to consider the role that these institutions have to play in regard to their policies of income and attention to diversity, so that society in general and particularly young people whose skills allow access to institutions can do so and comply with the respective studies curriculum in order to achieve their goals and achieve self-realization.

Key words: diversity, higher education; university 


\section{Introducción}

Actualmente, la diversidad enfrenta a la universidad a situaciones que parecen tender a obviar el crecimiento real de los estudiantes. Las instituciones universitarias tradicionalistas se fundamentaban en un pretendido modelo único de educación al que debían adaptarse los estudiantes independientemente de su procedencia, modelo que entró en decadencia por su cuestionamiento social y por no ofrecer a los estudiantes, alternativas efectivas para su adaptación a este medio tan complejo como lo es el medio universitario.

Además, la educación como derecho universal, constituye un proceso para el desarrollo de la dignificación y felicidad del ser humano. Es por ello que la educación como un sistema establece en sus leyes y normativas la garantía por parte del estado de una educación de calidad para todos, permanente, continua, integral, con equidad de género, igualdad de condiciones y oportunidades, derechos y deberes. Así mismo, es importante destacar que uno de los aspectos importantes en el sistema educativo es la prosecución de los estudios, esto quiere decir que el estado debe proporcionar la estructura necesaria, no solo para el ingreso a este sistema, sino también para su mantenimiento prolongado que les permita a las personas independientemente de su raza, sexo o credo alcanzar las metas propuestas en el pleno desarrollo de los individuos como profesionales, logrando así su autorrealización.

Como lo plantea Garabal (2015), en la actualidad se presenta un descenso considerable del alumnado en el acceso a la universidad, hecho que perjudica a la igualdad en el contexto educativo. Con base en datos de España, Garabal evidencia la existencia de dificultades en la realización de los estudios universitarios por parte del colectivo con diversidad funcional, y justifica la necesidad de llevar a cabo investigaciones en esta corriente. Otros autores como Lledó, Perandones \& Sánchez (2012), Rodríguez, Álvarez \& García-Ruiz (2014), abordan la problemática de la diversidad funcional en el contexto universitario.

Se hace necesario destacar que las instituciones universitarias iniciaron su filiación histórica en el Medioevo europeo y, a través de rupturas y continuidades, han mantenido una legitimidad social hasta el presente. Su importancia es relevante, al punto de generar expectativas y debates por su estado presente y las potencialidades de su evolución futura. Las universidades han existido antes que el estado nacional y seguramente sobrevivirá hasta el futuro, ya que la historia de la institución universitaria se despliega en la larga duración, desde la que se vislumbra la 
transferencia y evolución de los modelos más importantes del mundo moderno, así como, sus procesos de transformación y las tendencias de su evolución a futuro.

De allí que, se hace importante considerar el papel que estas instituciones les corresponde desempeñar en lo que respecta sus políticas de ingreso y atención a la diversidad, para que la sociedad en general y de manera particular los jóvenes cuyas competencias permitan su acceso a las instituciones universitarias puedan hacerlo y cumplir con los pensum de estudios respectivos con la finalidad de alcanzar sus metas y lograr su autorrealización.

\section{Etimología de la Palabra Diversidad}

La palabra diversidad viene del latín diversitas y este del participio diversus del verbo divertere (Girar en dirección opuesta), desde el punto de vista estructural está compuesta del prefijo Di que significa divergencia o separación múltiple y el verbo Vertere que significa verter, girar, dar vueltas y el sufijo Dad el cual indica cualidad de lo diverso o diferente que por ser diverso necesariamente ha de ser múltiple y abundante. La asociación de la idea de divertere y diversitas se debe a que cuando hay variedad se puede ver cosas diferentes, completamente opuestas. De lo anterior se puede deducir que la existencia de cosas variadas y disímiles giradas en direcciones opuestas, pueden generar entidades cada vez más variadas, transformadas y renovadas.

\section{El término diversidad, puede referirse a:}

- Diversidad biológica, un parámetro ecológico empleado en biología, es, según el Convenio Internacional sobre la Diversidad Biológica, el término por el que se hace referencia a la amplia variedad de seres vivos sobre la Tierra y los patrones naturales que la conforman.

- Diversidad sexual en relación a la sexualidad humana, entendida esta como el fenómeno por el cual una especie animal presenta individuos de diferentes sexos identidades sexuales y orientaciones sexuales.

- Diversidad Cultural, en cuanto a la riqueza cultural de un grupo humano, se refiere al grado de variación cultural, tanto a nivel mundial como en ciertas áreas, en las que existe interacción de diferentes culturas coexistentes.

- Diversidad linguística, en cuanto a la riqueza de lenguas, se refiere a una medida de la cantidad de lenguas existentes en un país o área geográfica.

- Diversidad Ecológica, en ecología el término diversidad ha designado tradicionalmente un parámetro de los ecosistemas (aunque se considera una propiedad emergente de la comunidad) que describe su variedad interna. 
- Diversidad Funcional, es un término alternativo al de discapacidad que ha comenzado a utilizarse en España por iniciativa de los propios afectados. El término fue propuesto en el Foro de Vida Independiente, en enero de 2005, y pretende sustituir a otros cuya semántica puede considerarse peyorativa.

Según Abbagnano (2010), el término diversidad se refiere a: "toda alteridad, diferencia o desemejanza. El término es más genérico que esos tres uno cualquiera de ellos o todos en conjunto. Puede indicar también la simple distinción numérica que se tiene cuando dos cosas no difieren en nada.” (p. 328), para los efectos de este documento la primera acepción del término es considerada con mayor articulación para lo que se desarrolla posteriormente ya que, en el presente trabajo se refiere a la diversidad como un elemento de las diferencias humanas individuales presentes en el recinto universitario. De allí que la diversidad pudiera estar representada por la negación de la identidad, es por esto que Wolff, la consideraba afirmando que son diferente las cosas que no pueden sustituirse una a otras, quedando sólidos los predicados que se atribuyen a una de ellas absolutamente o en determinada condición.

\section{Dimensión Histórica de la Universidad}

El término Universidad proviene del latín Universitas y significa Universo o totalidad, según Cárdenas (2004): “Totalidad de todo lo que es y se necesita conocer, totalidad de todo un género de cosas, totalidad colectiva, comunitaria o corporativa: Universitas Magistrorum y Universitas Studentium" (p.406), es una institución educativa de estudios y titulaciones académicas superiores de alto contenido universal, su objeto fundamental es formar de manera integral y profesional a los estudiantes, contribuir con el desarrollo de las ciencias, las artes, las humanidades y la cultura, poniéndose al servicio de la sociedad para fomentar y sustentar el desarrollo y progreso de las comunidades y el país.

Según López, Carrión y Bermúdez (1995). "Las raíces originarias del establecimiento universitario podríamos rastrearlas hasta antes del Medioevo" (p.76). A partir de allí se han realizado diversos recorridos por la dimensión histórica universitaria en el mundo. La universidad vista con una visión de universalidad ha reconstruido su enfoque y emerge en diferentes ámbitos, lo que Gustavo Rojas Bravo denomina "programas deseables" para las instituciones universitarias. Bravo (1999) “identificó en tres grandes grupos las principales tendencias y cambios, que a comienzo de la década de los setenta, eran considerados para la planeación del desarrollo universitario" (p.29), estos grupos a los cuales se refiere son los siguientes: 
a) Los cambios externos que ocurren fuera de la universidad y en los cuales se refleja el incremento de la complejidad del medio ambiente socioeconómico.

b) Cambios internos, que van a reflejar una vida interna de las universidades cada vez más complejas, y que deben abordar nuevas tareas para satisfacer, nuevas necesidades, intereses y demandas.

c) La redefinición de la relación entre la universidad y la sociedad, en la cual las universidades muestran e incrementan su rol socioeconómico.

Esta caracterización según lo planteado por Bravo, (1999) "Muestra la dirección deseada y previsible del cambio universitario, que con la promesa de la planeación se planteaba a las universidades en la década de los setenta y recoge los esfuerzos, tendencias e orientaciones acumuladas desde el final de la guerra" (p.31). Es importantes destacar que, en la actualidad hay elementos que se mantienen presentes y que representan estigmas del pasado de las universidades lo que conduce a un acto de auto reflexión en las universidades para reconsiderar los objetivos y propósitos de la institución, su rumbo y la red de relaciones sociales que incide sobre su actividad. Además, la innovación en las universidades y sistemas de educación superior trajo consigo una expansión histórica social lo cual generó universidades innovadoras de nueva creación.

Así pues, es necesario señalar que la universidad es un espacio multicultural, que aparece en todas las sociedades sirviendo de puente entre la formación sistemática terminal y los desarrollos que hacen avanzar la civilización. Para algunos actores esa relación cultural de la universidad tiene un carácter permanente y esencial, por esta razón el campus universitario es visto como una cristalización transhistórica no afectada por los cambios de la sociedad.

\section{La Diversidad desde el ámbito Universitario}

La diversidad como fenómeno socio - antropológico ha sido objeto de estudio permanente, sin embargo, ¿qué relación conceptual puede existir entre la universidad y la diversidad como elemento de interacción? y ¿qué elementos de adaptación pueden considerarse para la diversidad en este mundo tan complejo como lo es el mundo universitario? El cual, exige del sujeto que aprende una autovaloración que le permita avanzar hasta concluir de manera satisfactoria su carrera. 
La diversidad es un aspecto difícil de asumir en los diferentes elementos que componen la sociedad, en los ámbitos de la vida personal y social, en la familia, en el trabajo, pero principalmente en las instituciones universitarias genera un impacto importante como un fenómeno social y antropológico el cual representa un importante objeto de estudio. Todo esto se debe en gran medida a la comodidad con que los seres humanos aprecian todo aquello que les resulta familiar, en contraste a lo desconocido, a lo diferente, a lo diverso.

En articulación con lo antes expuesto, es necesario tomar en consideración los conceptos que desarrolla Maturana (1999), en los desafíos pedagógicos de la transformación educativa, principalmente si se pregunta ¿Qué es educar a una persona diversa con los retos que plantea el nuevo milenio?; Se considera importante tener una visión de la educación desde la óptica de este autor, la cual consiste en abrir espacios para la formación de individuos pertenecientes, a la diversidad, como seres que son punto de partida para las acciones; no es la oposición o la diferencia lo que hace a una persona un individuo, sino el poder hacer, lo que hace desde sí, aprende desde sí, discrepa desde sí. Así lo confirma Maturana (1999): “Como un ser centrado en sí mismo, no en la oposición, no en la diferencia, sino como el núcleo desde el cual hace lo que hace; bien o mal, no es el punto. Hace lo que hace. No está justificándose por ser. Simplemente es, en un espacio que acepta su ser" (p.137).

Partiendo de lo anterior, se puede afirmar que la diversidad es un enfoque educativo postmoderno y lo confirma Puigdellivol (1.998), al señalar que: "individualizar, compensar y promover igualdad de oportunidades que viene a considerar como relevante desde este punto de vista de las circunstancias de ser diferente" (p. 5). En este sentido, la universidad actual debe asumir un rol transformador, comprometiéndose con los estudiantes a facilitar la información necesaria y propiciar una autentica equidad en su camino a los diferentes niveles educativos.

En el 2do Congreso Internacional de Educación y Diversidad celebrado en España en el año (2001); se hace referencia a que todos los estudiantes tienen capacidades, intereses, expectativas, inteligencias, estilos de aprendizaje, motivaciones, intereses y necesidades, aptitudes, culturas, historias de vida diferentes, todo esto implica que cada uno de ellos, tengan también unas necesidades educativas propias y específicas, que requieren una atención pedagógica especializada que le permitan acceder al aprendizaje con igualdad de oportunidades para así disfrutar de una educación de calidad. Así pues, la atención a la diversidad en las universidades 
implica considerar a cada uno como individualidad tomando en cuenta, el género, la raza, el nivel socioeconómico y su cultura en general.

Lo expuesto anteriormente permite inferir que, la diversidad abarca muchas formas de diferencia, entre las cuales se pueden destacar:

- $\quad$ La diversidad que se aleja un poco de la norma

- $\quad$ El género, el interés, la capacidad, la aptitud y la motivación del estudiante por aprender

- $\quad$ Los estudiantes con necesidades educativas especiales

- $\quad$ La diversidad que es fruto de una diversidad multicultural, multilingüe y de la integración de minorías étnicas y religiosas.

Partiendo de lo anterior, Bauman (2004), señala en relación a la diferencia como elemento medular de la diversidad y a la cultura como aspecto inherente al ser humano que: "La nueva indiferencia a la diferencia es teorizada como reconocimiento del 'pluralismo cultural', y la política informada y sustentada por esta teoría se llama a veces 'multiculturalismo'. (p. 107).

Según Martinez, (2006), el hombre se educa y se transforma en su contexto, siendo la sociedad el espacio educador más efectivo y globalizante. Partiendo de esta afirmación, La educación y la cultura son productos sociales que se implican mutuamente cuando esta correspondencia ocurre de manera permanente y sistemática, la sociedad se enriquece y la formación de los individuos con diversidad funcional es más sólida, siendo el contexto, el concepto de educación, el conocimiento, la capacitación, la actualización, los procesos de cambio, la experiencia y lo sociocultural, los elementos considerados como el principio del proceso educativo.

vale la pena reseñar, antes de abordar las reflexiones finales, que en el contexto ecuatoriano no son muchas las investigaciones que se encuentran en la literatura del tema; sin embargo, se destacan los trabajos de Delgado (2016), quien presenta una Propuesta de aplicación del enfoque de Educación Inclusiva en Educación Media y sus proyecciones a la Educación Superior; Espinosa, Gómez y Cañedo (2012), quienes plantean el Acceso y la Retención en la Educación Superior de Estudiantes con Discapacidad en Ecuador; y Jara, Melero y Guichot (2015), quienes analizan la inclusión socioeducativa, perspectivas y desafíos, entre otros autores.

Reflexiones finales

La sociedad actual, se ha construido a partir de la pluralidad del pensamiento, de expresiones culturales y por la diversidad de manifestaciones sociales de los grupos humanos pertenecientes a esta. Así pues, la sociedad considera las instituciones educativas como única opción para el 
desarrollo del ser humano desde el punto de vista científico, tecnológico, económico y social. Por consiguiente, el hombre se educa conforme a dos procesos fundamentales; por lo que vive en su dia a dia (proceso social), y por lo que aprende al adquirir experiencias de sus necesidades contextuales (proceso individual).

Es necesario, en estos tiempos de cambios tomar conciencia de los mismos en la sociedad y como todos sus integrantes pueden participar, para que se dé una verdadera transformación positiva del conocimiento lo cual es producto de la influencia y la acción del hombre. De allí que, se requiere de una actualización del conocimiento en la universidad, lo cual traerá como consecuencia que ésta se convierta en espacios donde se promueva un proceso permanente de formación docente, concebido este como un proceso motor y facilitador de aprendizajes en un contexto social de experiencias educativas nuevas.

La educación para la diversidad en las universidades requiere docentes con un amplio conocimiento y dominio especializado, de lo que denomina Alamis (2001), un campo específico del quehacer educativo. Además, que sea creativo, responsable, gestor de proyectos y programas de innovación educativa, que investigue y genere conocimientos todo esto llevado a la práctica diaria en su quehacer universitario y no olvidar que la acción de educar es una tarea humanizadora donde se contempla, la orientación, la guía, la ayuda del otro, para el desarrollo de sus potencialidades y sus capacidades para recibir y forjar valores. En la figura 1, se muestra la educación de la persona con diversidad funcional como producto de multivariables contextuales que le influirán de manera positiva o negativa en su comportamiento, manera de pensar, actuar e interpretar la realidad, además en su desarrollo personal y profesional.

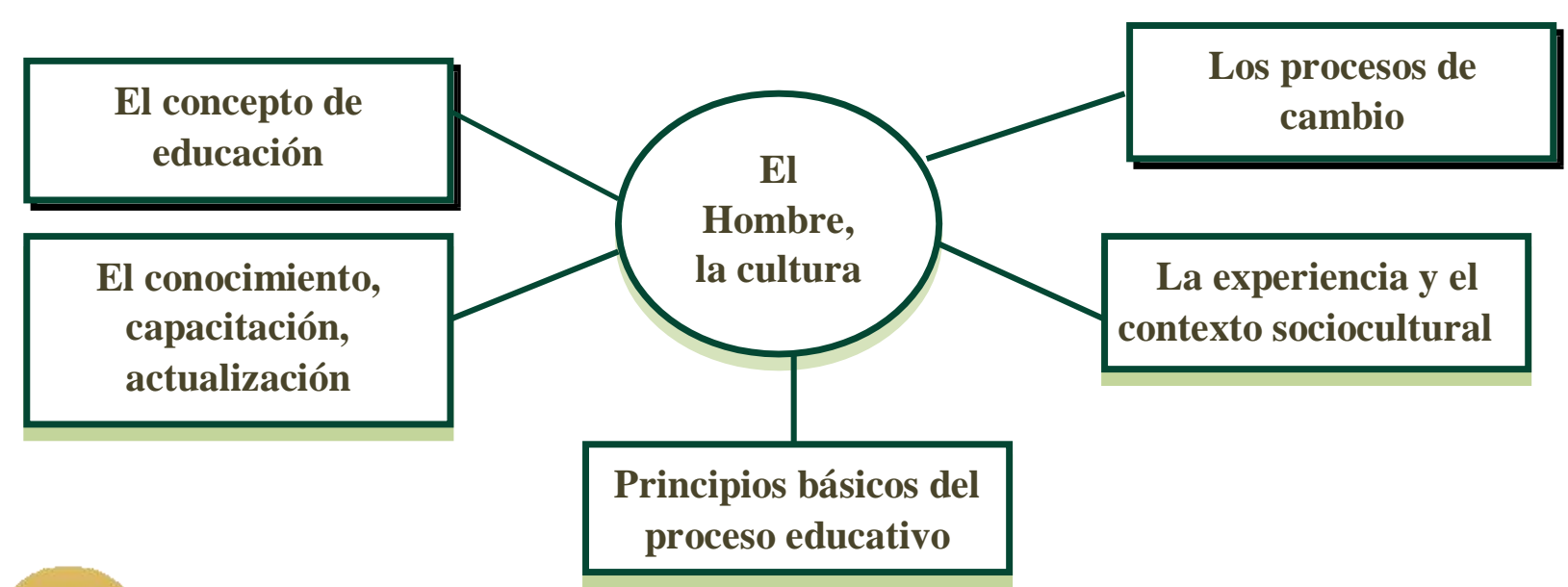


Figura 1.- La educación de la persona con diversidad funcional

Fuente: Alamis (2001, p.15)

En este sentido, al mirar la diversidad se ven las semejanzas y al ver las semejanzas uno se puede preguntar por su origen, pertenecemos a una diversificación de modos de vida, somos y además, somos seres que vivimos en el acto de conversar; entonces, nuestro espacio relacional consiste en redes de conversaciones que constituyen la cultura donde nos desarrollamos. Todos estos aspectos sumados a lo que Maturana llama biología del amar son piezas que engranan perfectamente en el rompecabezas de la diversidad en el ámbito universitario, ya que todos esos aspectos biológicos, sociales, y psicológicos que conforman al ser diverso le permitirán estructurar más y mejor su capacidad de adaptación a cualquier entorno incluyendo la universidad.

\section{Referencias Bibliográficas}

Alamis, A. (2001). El saber hacer en la profesión docente. Formación profesional en la práctica docente. México: Trillas

Bauman, Z. (2004). Trabajo, Consumismo y Nuevos Pobres. Barcelona: Gedisa.

Bravo, F. (1999). Universidad, Ciencia y Conciencia, Conocimiento y Universidad. Valencia, Venezuela: Universidad de Carabobo.

Cárdenas, C. (2004). Alternativas de la Educación Superior CENDES, No 2 y 3. Universidad Central de Venezuela.

Congreso Internacional de Educación y Diversidad España (2001). Selección de ponencias y comunidades. Año 2001 actas (Madrid)

Delgado, K. (2016). Propuesta de aplicación del enfoque de Educación Inclusiva en Educación Media y sus proyecciones a la Educación Superior. RECUS. Revista Electrónica Cooperación Universidad - $\quad$ Sociedad, 1(3), 27-33. Recuperado de: http://revistas.utm.edu.ec/index.php/Recus/article/download/57/45/ 
Escandell, M. \& Rodríguez, A. (2008). Avances legales sobre diversidad en la universidad. International Journal of Developmental and Educational Psychology, 20(1), 137-146. Recuperado de: http://infad.eu/RevistaINFAD/2008/n1/volumen3/INFAD_010320_137-146.pdf

Espinosa, C.; Gómez, V. y Cañedo, C. (2012). El Acceso y la Retención en la Educación Superior de Estudiantes con Discapacidad en Ecuador. Formación Universitaria, 5(6), 27-38. DOI: http://dx.doi.org/10.4067/S0718-50062012000600004

Garabal, J. (2015). Universidad y diversidad funcional: aproximación a la inclusión en el ámbito universitario. Revista de Estudios e Investigación en Psicología y Educación, 8, 1-6. DOI: https://doi.org/10.17979/reipe.2015.0.08.218

Jara, R.; Melero, N. y Guichot, E. (2015). Inclusión socioeducativa, perspectivas y desafíos: Universidad Politécnica Salesiana del Ecuador y Universidad de Sevilla-España. ALTERIDAD. Revista de Educación, 10(2), 164-179. DOI: http://dx.doi.org/10.17163/alt.v10n2.2015.03

Lledó, A.; Perandones, T. \& Sánchez, F. (2012). La diversidad en la universidad: una cuestión pendiente. International Journal of Developmental and Educational Psychology, 3(1), 147-153. Recuperado de: http://www.redalyc.org/pdf/3498/349832338014.pdf

López, F., Carrión, R., Bermúdez, L. (1.995) El Reordenamiento de la Universidad Autónoma. Cuadernos del Debate No 1 . CRU. Universidad de Carabobo. Valencia - Venezuela.

Maturana, H. (1999). Transformación en la convivencia. Santiago de Chile: Dolmen ediciones.

Martínez, E. (2006). Una Educación Integral a Través de Estrategias de Enseñanza y Aprendizaje para el Desarrollo Cognoscitivo, Metacognoscitivo y Afectivo en la Formación Docente. Tesis Doctoral Universidad de Carabobo.

Puigdellivol (1.998), La educación especial en la escuela integrada: Una perspectiva desde la diversidad. Barcelona, España: Editorial GRAO.

Rodríguez, A.; Álvarez, E. \& García-Ruiz, R. (2014). La atención a la diversidad en la universidad: el valor de las actitudes. Revista Española de Orientación y Psicopedagogía, 25(1), 44-61. Recuperado de: http://www.redalyc.org/pdf/3382/338232571004.pdf 